
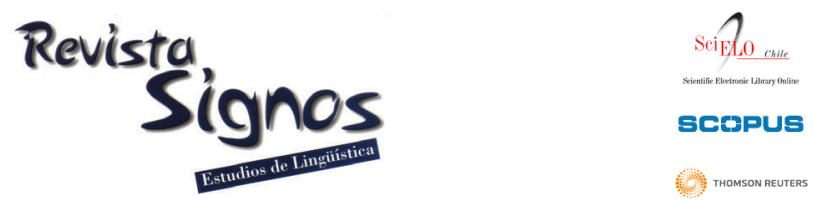

\title{
Argumentación y estandarización lingüística: Creencias normativas en el 'Diccionario de chilenismos' (1875) de Zorobabel Rodríguez ${ }^{1}$
}

\section{Argumentation and linguistic standardization: Normative beliefs in Zorobabel Rodríguez's Diccionario de chilenismos (1875)}

Tania Avilés

Universidad de Chile

ChiLe

taviles@ug.uchile.cl
Darío Rojas

Universidad de Chile

Chile

darioroj@u.uchile.cl

Recibido: 22-VIII-2012 / Aceptado: 7-VIII-2013

\section{Resumen}

En el presente estudio, enmarcado en la historiografía de las ideologías lingüísticas, describimos cuáles eran las creencias que funcionaban como criterios normativos para la estandarización lingüística en el Chile de fines del siglo XIX, tal como se encuentran representadas en el 'Diccionario de chilenismos' (1875) del abogado y político chileno Zorobabel Rodríguez. Efectuamos esta descripción a través del análisis del discurso argumentativo contenido en una muestra de entradas del diccionario, a las cuales aplicamos el modelo de análisis de Toulmin. Mediante este análisis, de enfoque cualitativo, identificamos conclusiones, datos y garantías, y consideramos estas últimas como reveladoras de las creencias normativas de Rodríguez. Encontramos siete creencias normativas en la muestra, que revelan un modelo ideal de lengua española congruente con la ideología monoglósica y compatible con las ideas lingüísticas racionalistas y propeninsulares de los unionistas chilenos.

Palabras Clave: Historiografía lingüística, ideologías lingüísticas, estandarización lingüística, argumentación, diccionarios de provincialismos. 


\begin{abstract}
This study, framed in the historiography of language ideologies, describes the beliefs that worked as the normative criteria in linguistic standardization during late 19th century in Chile, as observed in the Diccionario de chilenismos (1875) written by the Chilean lawyer and politician Zorobabel Rodríguez. To achieve this, we used Toulmin's analytic model to analyze the argumentative discourse from a sample of entries from this dictionary. Using qualitative analysis, we identified claims, data, and warrants. We considered warrants as revealing of Rodríguez's normative beliefs. We found seven beliefs in our sample, which show an idea of the Spanish language congruent with the monoglossic language ideology and compatible with the rationalist and pro-Spain linguistic ideas of Chilean unionistas.
\end{abstract}

Key Words: Linguistic historiography, language ideologies, linguistic standardization, argumentation, dictionaries of provincialisms.

\title{
INTRODUCCIÓN
}

Varios estudios pertenecientes al ámbito de la historia de la lexicografía (Matus, 1994; Castillo, 1995; Becerra, Castro \& Garrido, 2007; Chávez, 2009) han abordado como objeto de análisis el 'Diccionario de chilenismos', del abogado y político católico liberal chileno Zorobabel Rodríguez (1875). Estos estudiosos han puesto énfasis en el desajuste que el 'Diccionario de chilenismos' presenta respecto de las técnicas lexicográficas modernas, por ejemplo, en la baja formalización de su microestructura o la subjetividad de sus definiciones, o bien han resaltado su carácter normativo considerándolo como manifestación de su carencia de método científico. Sin embargo, las ideologías y actitudes lingüísticas que aparecen manifestadas en esta obra han concitado solo un interés secundario. Por otra parte, cuando este problema ha sido abordado (Matus, 1994; Chávez, 2010), solo se han considerado los componentes lexicográficos paratextuales (prólogos), desaprovechando el abundante discurso contenido en cada una de las entradas o artículos lexicográficos, donde además se encuentra la "evidencia más confiable" para este tipo de investigaciones (Coleman \& Ogilvie, 2009: 2). En consecuencia, aún está pendiente el estudio exhaustivo de las ideas y actitudes sobre el lenguaje expresadas o implicadas en el 'Diccionario de chilenismos' (1875), así como en los demás diccionarios de este tipo publicados en Chile durante la segunda mitad del siglo XIX y comienzos del siglo XX. El conocimiento de estas ideas y actitudes es importante porque el contexto sociolingüístico-histórico de esta obra corresponde a un proceso de estandarización, en el que el aspecto ideológico ocupa un lugar central.

El objetivo del presente estudio es describir, en el marco de la historiografía de las ideologías lingüísticas, cuáles son las creencias lingüísticas normativas que articulan el discurso argumentativo contenido en el diccionario de Rodríguez. Realizaremos el análisis sobre la base de una muestra de entradas lexicográficas que contienen segmentos 
relativamente extensos de discurso argumentativo, en los que el autor del repertorio sopesa distintas razones para considerar aceptables o inaceptables algunos de los usos que diferenciaban al español de Chile del español peninsular. En la línea del análisis del discurso orientado al contenido, empleado para indagar en modelos culturales del lenguaje (Preston, 2011), usaremos como herramienta el modelo de análisis funcional y contextual del discurso argumentativo propuesto por Toulmin (2007). Con la ayuda de esta herramienta, identificaremos las garantías, correspondientes a las creencias de tipo normativo sobre el idioma, que autorizan el encadenamiento entre los datos y las conclusiones entregadas en cada una de las entradas del diccionario analizadas. A partir de estas creencias, intentaremos reconstruir la ideología lingüística que se manifiesta en el texto analizado ${ }^{3}$.

\section{Marco conceptual}

\subsection{Ideologías y actitudes lingüísticas}

La orientación teórica que agrupa a los estudios de ideologías lingüísticas ha mostrado ser una fuente rica en recursos conceptuales y metodológicos para aproximarse a la relación entre lenguaje y cultura y ha tenido un desarrollo sostenido y creciente a partir de la década de los 70 (Kroskrity, 2010). Aunque el estudio lingüístico de las ideologías suele asociarse principalmente al análisis crítico del discurso, esta perspectiva suele concentrarse más bien en las ideologías en el lenguaje, y no tanto en las ideologías sobre el lenguaje, que constituyen nuestro foco de interés.

Entendemos por 'ideología lingüística' el conjunto de creencias y concepciones acerca del lenguaje, planteadas de manera explícita o manifestadas implícitamente en prácticas comunicativas, que sirven a una comunidad para racionalizar el uso lingüístico y que a menudo responden a sus intereses políticos y económicos, sea los de la totalidad de sus miembros o los de grupos sociales determinados (Kroskrity, 2010). Las ideologías de este tipo, además, manifiestan la relación mental que, en la percepción de los hablantes, el lenguaje tiene con los valores estéticos, morales y epistemológicos de la comunidad (Woolard, 1998). Otros rasgos definitorios de las ideologías lingüísticas son que los miembros de la comunidad pueden tener diversos grados de conciencia de ellas (Kroskity, 2010) y que no necesariamente corresponden a sistemas coherentes de significación, pues pueden manifestarse como fragmentarias e internamente contradictorias (Woolard, 1998), e incluso múltiples dentro de un individuo o una comunidad, razón por la cual Kroskrity (2010) prefiere hablar de ellas en plural. Adoptamos, en nuestro estudio, una postura 'neutral' o 'ideacional' respecto del estudio de ideologías, por oposición a las posturas 'críticas' o 'negativas' de inspiración principalmente marxista (Woolard, 1998).

Según Kroskrity (2010), este campo de estudio problematiza simultáneamente la conciencia metalingüística de los hablantes y su posición evaluativa respecto de su instrumento comunicativo. Al considerar el componente evaluativo, el estudio de 
las ideologías se vincula estrechamente con el de las actitudes lingüísticas (Garrett, 2010). El concepto de 'actitud' proviene de la sicología social, ámbito en que se define como la "tendencia sicológica que se expresa mediante la evaluación de una entidad particular con algún grado de aprobación o desaprobación" (Albarracín, Zanna, Johnson \& Kumkale, 2005: 4). Lo específico de una actitud lingüística es que la entidad particular evaluada corresponde a un rasgo lingüístico o una variedad lingüística completa. Usualmente las actitudes son concebidas en términos de una estructura tripartita: creencias (componente cognitivo), emociones (componente afectivo) y conductas (componente conductual) (Bizer, 2004).

El elemento cognitivo de las actitudes constituye el punto de encuentro entre estas y las ideologías lingüísticas. Maio, Olson, Bernard y Luke (2006) señalan que ideologías y actitudes, aunque comparten el carácter evaluativo y subjetivo, se diferencian por el grado de abstracción: mientras que las ideologías comprenden sistemas generales de creencias, valores y actitudes, y por lo tanto tienen un mayor grado de abstracción, las actitudes suelen estar enfocadas en objetos específicos y de carácter más concreto. Por otra parte, ambos constructos se encuentran interrelacionados, de modo que el nivel más abstracto (ideología) influye en el nivel más concreto (actitud). Es de esperar, entonces, que distintas ideologías lingüísticas tengan reflejo en distintas actitudes hacia un mismo objeto de tipo lingüístico.

Entre los desarrollos recientes de los estudios lingüístico-ideológicos se encuentra la historiografía de las ideologías lingüísticas (Blommaert, 1999; Kroskrity, 2010), que se ocupa del estudio de cómo se conforman, reproducen y transforman a lo largo del tiempo estos conjuntos de creencias acerca del lenguaje. Esta corriente de estudios pone énfasis en el carácter de 'debates lingüísticos' que suelen adquirir estos procesos históricos, es decir, debates en los que "el lenguaje es el tema central, un motivo, un objetivo, y en los que las ideologías lingüísticas se forman, enmiendan o refuerzan" (Blommaert, 1999: 1), y que además tienen por marco "determinados periodos históricos y [...] procesos políticos y culturales específicos" (De los Heros, 2012: 24) o, dicho de otro modo, "forman parte de procesos sociopolíticos más generales" (Blommaert, 1999: 2; también Coupland \& Jaworski, 2004).

En consecuencia, el estudio historiográfico de las ideologías sobre el lenguaje requiere, por un lado, la consideración del discurso argumentativo desarrollado en los textos por analizar, por la importancia que los debates lingüísticos tienen en su constitución histórica. Es por esto que hemos escogido una perspectiva de análisis centrada en el discurso argumentativo. Por otro lado, requiere la consideración detallada del contexto específico de producción de los textos. En el apartado siguiente, examinaremos este contexto, correspondiente al proceso de estandarización lingüística iniciado en gran parte de Hispanoamérica a lo largo del siglo xix, a raíz de las Independencias, en el cual los diccionarios de provincialismos, como el de Rodríguez, cumplieron una función instrumental. 


\subsection{El contexto de producción: Estandarización y diccionarios de provincialismos en la Hispanoamérica decimonónica}

La obra de Rodríguez, publicada en 1875, tiene por contexto de producción la consolidación de los procesos políticos e ideológicos iniciados a raíz de las independencias de gran parte de las naciones americanas respecto de la corona española. En Chile, el proceso de emancipación política transcurre entre 1810, año de la Primera Junta Nacional de Gobierno, y 1823, año en que Bernardo O'Higgins abdica del cargo de Director Supremo. Como correlato de este proceso político, la comunidad hispanohablante chilena, según Matus, Dargham y Samaniego (1992), a fines del siglo XIX ya había entrado en un proceso de estandarización circunstanciada, es decir, un proceso que comienza a transformar una variedad vernácula (el español de Chile) en estándar nacional a través de la imposición de una norma, bajo la influencia de factores de prestigio no lingüístico que hicieron del español local la variedad escogida sin mayor discusión frente a eventuales opciones como lenguas indígenas o extranjeras (Joseph, 1987). Este proceso se caracteriza por hitos como la publicación de obras que se proponían codificar la lengua española (como la Gramática de Andrés Bello, de 1847) y la oficialización de la lengua española en la enseñanza chilena, entre otros. El papel central que las ideologías lingüísticas desempeñan en los procesos de estandarización es destacado por Inoue (2006: 121), quien señala que el estudio de estos procesos "no tiene tanto que ver con el lenguaje en sí en cuanto función y estructura como con las ideologías y hegemonías lingüísticas"; es decir, con cómo ocurre el reconocimiento social de un estándar y qué factores sociales, políticos e históricos hacen posible este proceso. Por otra parte, es reconocido que los procesos de estandarización lingüística forman parte, en el nivel simbólico, de los procesos de conformación de las naciones modernas (Blommaert, 1999), específicamente, de su 'invención metadiscursiva' (Metzeltin, 2011).

En el clima intelectual de los movimientos independentistas hispanoamericanos, el idioma español se convirtió en uno de los objetos de reflexión más significativos para las élites ilustradas, pues presentaba, simultánea y conflictivamente, los caracteres de, por un lado, tradición heredada de los antiguos dominadores y, por otro, potencial vehículo ideal y natural para la participación en la vida cívica de las nuevas naciones y para la difusión de las ideas entre sus integrantes. De esta manera, el siglo xix hispanoamericano, especialmente a partir del segundo cuarto del siglo, atestiguó el surgimiento de actitudes diversas hacia la lengua española. Había quienes, imbuidos de un pensamiento nacionalista, renegaban de todo lo español que se les había heredado, mientras otros consideraron al español como símbolo identitario e integrador e insistieron en su cultivo institucional a nivel internacional (Lara, 2009a).

En Chile, los intelectuales se adhirieron principalmente bien al grupo que Quesada (2002) denomina 'separatistas', cuyo mejor representante es Domingo Faustino Sarmiento, o bien a los 'unionistas', que seguían fundamentalmente 
las ideas de Andrés Bello. Los 'separatistas' planteaban una escisión lingüísticoideológica respecto de España, lo que conllevaba valorizar la diferencia idiomática con la metrópoli y de esta manera reforzar la autonomía identitaria de las nuevas naciones, es decir, deseaban que la autoridad idiomática estuviera circunscrita al país. Sin embargo, fueron los 'unionistas', como en otras naciones americanas, quienes triunfaron en esta pugna ideológica gracias a su influencia política y cultural. Por esta razón, les fue posible aplicar sus ideas mediante una política lingüística de tipo prescriptivo apoyada de manera oficial por el Gobierno y materializada en numerosas obras (gramáticas y diccionarios) destinadas a la corrección de los hábitos idiomáticos que iban en detrimento de la unidad y casticidad de la lengua española en América.

En su aparato ideológico ocupaba un lugar central la unidad del idioma, que era considerada importante porque evitaría una indeseable fragmentación dialectal de la lengua española en Hispanoamérica, análoga a la que había experimentado el latín al caer el Imperio romano en el siglo $\mathrm{V}$ d. C. e iniciarse una era que las naciones europeas veían con malos ojos por su oscuridad cultural, y que los americanos no querían ver replicada, por lo tanto, en sus nuevas naciones. Su visión, por lo tanto, no podía circunscribir la autoridad idiomática a los límites de la nación: para ellos se trataba de un problema de la comunidad hispanohablante en su conjunto.

Por otra parte, la preocupación por la unidad condujo fácilmente a una gran estimación de la corrección idiomática: la lengua se transformó en un ideal platónico (el español correcto, ejemplar, castizo) sobre cuyo trasfondo eran evaluadas las conductas idiomáticas de los individuos. Las únicas formas lingüísticas que adquieren legitimidad, en este trasfondo, son las 'correctas', es decir, las que se ajustan al modelo platónico de lengua, y se entiende que la unidad de la lengua se conservará solo en la medida en que la conducta idiomática de los miembros de la comunidad tienda hacia este ideal. El modelo de español (el 'español correcto') de los unionistas chilenos tenía un marcado tinte peninsular. Por ejemplo, a pesar de que Bello defendía, de palabra, las particularidades lingüísticas americanas, pensaba, paradójicamente, que los chilenos (y americanos) debían aprender a distinguir en la pronunciación entre la sibilante dental y la interdental, como los españoles (Moré, 2002).

El diccionario de Rodríguez se publica cuando esta actitud favorable al español peninsular y negativa hacia lo provincial ya estaba bastante arraigada en los medios cultivados chilenos, y especialmente entre los sectores más conservadores. Su multifacético autor (1839-1901), novelista, poeta, parlamentario, abogado (no titulado), profesor y periodista, fue uno de los representantes más notables de la intelectualidad conservadora de la segunda mitad del siglo xix. Sin embargo, no era un conservador 'puro': su propuesta política aunaba catolicismo y liberalismo, en cuanto defendía las libertades individuales frente al autoritarismo estatal, así como las libertades económicas (Correa, 1997). El propósito de su 'Diccionario', según el mismo lo declara, es proporcionar "un fácil medio de evitar los errores más comunes que, hablando o 
escribiendo, se cometen en nuestro país en materia de lenguaje" (Rodríguez, (1979 [1875]): viii). Para esto, recoge aproximadamente 1100 voces, comentadas bajo la modalidad de diccionario (por orden alfabético), precedidas de un prólogo en que explicita la finalidad de su obra. Por su propósito normativo y finalidad pedagógica, puede considerarse que representa fielmente el clima de opinión de la lexicografía precientífica chilena (Matus, 1994), y, sin duda, por su carácter pionero, fue muy influyente en las reflexiones del lenguaje que vendrían en las décadas siguientes. Para mostrarlo, basta con citar la publicación de varios trabajos posteriores dedicados únicamente a discutir su contenido: Blanco (1875), Paulsen (1876) y Solar (1876). Al momento de su muerte, Rodríguez se encontraba preparando una segunda edición del 'Diccionario', cuyos borradores probablemente se perdieron en un incendio en su casa de Valparaíso (Castillo, 1995).

Rojas (2010), siguiendo la visión pragmático-discursiva del diccionario de Lara (1997), señala que los diccionarios de provincialismos chilenos, como muchos otros publicados en Hispanoamérica en las décadas anteriores (Haensch, 2000), servían como vehículo discursivo para la evaluación de usos lingüísticos provinciales con miras a su incorporación al español estándar, y no meramente para informar acerca del significado de las palabras, como sucede en el caso de los diccionarios monolingües. Es decir, los diccionarios de provincialismos eran herramientas de estandarización lingüística. En cuanto obras normativas, pretendían corregir las (supuestas) incorrecciones del español chileno y americano, determinadas por su grado de diferencia respecto del español codificado en las obras académicas, de marcado tinte peninsular.

De acuerdo con la propuesta de Rojas (2010), la evaluación, en estos diccionarios, afecta a un uso lingüístico putativamente incorrecto (por ejemplo, porque es un provincialismo o porque otros autores anteriores lo han señalado), carácter respecto del cual se hacen 'comprobaciones' o 'refutaciones'. Si el autor considera haber comprobado su incorrección, mediante una argumentación o de manera implícita, señala cuál sería el equivalente correcto. Cuando refuta la incorrección, se ocupa de señalar cuáles son las razones que fundamentan su refutación. De este modo, el artículo lexicográfico de un diccionario de provincialismos típicamente adopta la configuración del Esquema 1, que expresa su estructura informativa: 


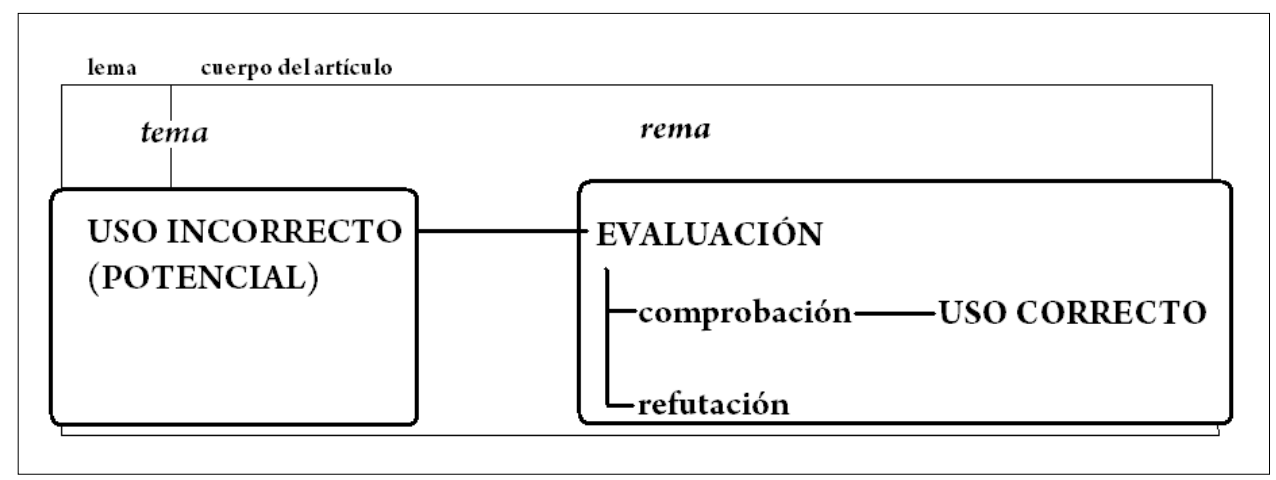

Cuadro 1. Forma esquemática del artículo lexicográfico como acto directivo (Rojas, 2010).

El espacio discursivo de la evaluación es fundamental para nuestro estudio, porque es allí donde Rodríguez suele desarrollar un amplio aparato argumentativo que revela sus creencias normativas acerca de la lengua, y que permite, por tanto, acceder a su ideología lingüística. En cuanto espacio en que se desarrolla la argumentación, además, es en él donde se desarrolla el debate lingüístico-ideológico del que habla Blommaert (1999).

Para abordar el análisis de estas creencias, tal como se manifiestan en estas argumentaciones, emplearemos el modelo de análisis del discurso argumentativo Toulmin (2007), que explicaremos someramente a continuación.

\subsection{La estructura de la argumentación según Toulmin}

Desde la lógica aplicada, Toulmin propuso en 1958 que el uso argumentativo del lenguaje se produce cuando las emisiones lingüísticas tienen éxito o fracasan a partir de su apoyo en sucesivos argumentos o pruebas. Por tanto, en su concepción, la argumentación refiere a la actividad de "plantear pretensiones, someterlas a debate, producir razones para respaldarlas, criticar esas razones y refutar esas críticas, etc." (Marafioti, 2005: 14). Para Toulmin (2007), los argumentos racionales, en general, deben analizarse de acuerdo con una serie compleja de categorías, a las cuales denomina 'conclusión' (C), 'datos' (D), 'garantía' (G), 'modalizadores' (M), 'condiciones de refutación’ (E) y ‘respaldo’ (R).

La conclusión corresponde a la aseveración realizada por un hablante, con la cual se ha comprometido. Los datos son aquellos hechos de que el hablante dispone para apoyar o justificar su aseveración, presentándolos como la base sobre la que esta descansa; es decir, son elementos justificatorios. Las garantías, por su parte, son aquellas reglas, principios, enunciados, etc., de carácter hipotético y general, que sirven de fundamento para formular la inferencia que permitirá el encadenamiento de los datos con la conclusión. Tanto los modalizadores como las restricciones o 
condiciones de refutación suponen un comentario implícito a la importancia de la garantía: los primeros indican la fuerza conferida por la garantía en el paso adoptado, mientras que las segundas apuntan las circunstancias en que la autoridad general de la garantía ha de dejarse a un lado. Finalmente, el respaldo o soporte corresponde a aquellas certezas sin las cuales las propias garantías carecerían de autoridad o vigencia, por lo que funcionan como fundamento de aquellas.

En nuestro análisis, solo consideramos los elementos C, D y G, es decir, las conclusiones, los datos y las garantías. Tomaremos estas últimas como reveladoras de las creencias normativas que articulan la ideología lingüística de Rodríguez.

\section{Metodología}

Nuestra investigación tiene un alcance descriptivo y un enfoque cualitativo. Analizamos una muestra de 200 entradas lexicográficas en que se desarrolla discurso argumentativo, seleccionadas mediante muestreo intencionado de entre las aproximadamente 1100 monografías ${ }^{4}$ que componen el total de la obra analizada (recuérdese que no todas ellas contienen discurso argumentativo, de modo que nuestro universo de muestreo es menor). Las entradas se encuentran repartidas entre todos los segmentos alfabéticos del diccionario. Las entradas analizadas se listan en el Anexo.

En nuestro análisis, primero identificamos el tipo de evaluación que se realizaba en cada artículo ('comprobación' o ‘refutación’). Luego, identificamos la conclusión, relacionada directamente con el tipo de evaluación: una comprobación corresponde a la conclusión implícita 'Esta palabra es incorrecta / No debe usarse esta palabra', y una refutación a 'Esta palabra es correcta / Puede usarse esta palabra' (Rojas, 2010). A veces hay elementos explícitos que permiten identificar fácilmente la conclusión, pero en buena parte de los artículos del diccionario el autor, en apariencia, se limita a describir el significado de un término. Sin embargo, en estos casos aplicamos el siguiente razonamiento. Rojas (2010) señala que las comprobaciones son más frecuentes que las refutaciones, y por lo tanto se encuentran más propensas a sufrir condensación textual (por economía de espacio). Esto se debe, a su vez, a que el macropropósito comunicativo del texto, declarado abiertamente en sus secciones preliminares, es listar provincialismos chilenos para censurar su uso. Entonces, asumimos que, si el autor simplemente 'muestra' un provincialismo chileno, el lector competente de la época interpretaría que este uso debe evitarse (véanse ejemplos en la nota 14 de Rojas, 2010).

A continuación, identificamos el dato y la garantía del argumento de cada artículo lexicográfico. Clasificamos los datos de acuerdo con clases que agrupaban datos similares en los artículos analizados. Finalmente, a partir de estas clases de datos, identificamos las garantías asociadas a ellas. 
El foco de nuestro análisis fue identificar clases de datos, con el fin de llegar a un nivel de generalidad suficiente como para llegar a inferir cuáles eran las garantías.

\section{Presentación y análisis de resultados}

Rodríguez recurre a cinco clases de datos en las comprobaciones y cuatro en las refutaciones, que presentamos esquemáticamente en la Tabla 1:

Tabla 1. Clases de datos en comprobaciones y refutaciones.

\begin{tabular}{|c|}
\hline Comprobaciones: "Es censurable porque... \\
\hline 1. “... está asociado principalmente al vulgo" \\
\hline 2. "... el $D R A E$ ya registra otro término equivalente que no es provincialismo" \\
\hline 3. "... no ha sido usado por autores literarios españoles" \\
\hline 4. "... es un extranjerismo sustituible por un término castizo" \\
\hline 5. “... es ajeno a las reglas gramaticales del idioma" \\
\hline Refutaciones: "Es aceptable porque... \\
\hline 1. “... lo registra el $D R A E$ y en realidad no es provincial" \\
\hline 2. "... lo usan autoridades literarias (españolas o americanas)" \\
\hline 3. "... satisface una necesidad denominativa" \\
\hline 4. "... se ajusta a las reglas gramaticales del idioma" \\
\hline
\end{tabular}

A continuación, explicaremos e ilustraremos con un ejemplo cada una de estas clases de datos y, en la discusión, intentaremos develar las garantías que les subyacen, las que, como señalamos al principio, reflejan las creencias lingüísticas normativas que propugna el autor del diccionario para el español de fines de siglo XIX. En adelante, escribiremos los enunciados que reflejan estas clases de datos en versalitas y las garantías en versalitas y negritas.

\subsection{Clases de datos en las comprobaciones}

\subsubsection{ES CENSURABLE PORQUE ESTÁ ASOCIADO PRINCIPALMENTE AL VULGO}

En primer lugar, Rodríguez censura los usos lingüísticos provinciales propios del 'vulgo'en el español de Chile de fines de siglo xix. Para el autor, el vulgo lo constituye aquella parte de la sociedad chilena que tiene escasa o nula instrucción formal. Por ejemplo, en la entrada de 'contra', Rodríguez concluye implícitamente que el uso de 'contra' por 'antídoto' es censurable. Para justificar su aseveración, señala como dato que este es un uso propio del vulgo chileno, que practica esta 'mala costumbre' idiomática, al igual que, aparentemente, el vulgo colombiano. Como respaldo de esta última parte del dato (el uso provincial colombiano), cita un ejemplo del vocabulario de la novela María de Jorge Isaacs (1867). Sin embargo, no aporta respaldo de que lo usa el vulgo chileno, con lo cual se entiende que se basa en su propio conocimiento de la realidad idiomática chilena. 
Cabe mencionar que Rodríguez no se refiere a que estos usos sean exclusivos del vulgo, sino a que se oyen principalmente en boca de este sector de la población, a pesar de que se pueden oír a veces también entre gente educada. Se trata, entonces, de una cuestión de uso preferente entre ciertos estratos, y no de diferencias absolutas. Por ejemplo, en la monografía sobre 'mono' señala que este término se usa “entre la jente zafia, aunque lo usa tambien la jente culta, pero no es para ésta tan socorrida voz como para aquélla” (Rodríguez, 1979 [1875]: 321).

\subsubsection{ES CENSURABLE PORQUE EL DRAE YA REGISTRA OTRO TÉRMINO EQUIVALENTE QUE NO ES PROVINCIALISMO}

En segundo lugar, Rodríguez cita como argumento de autoridad al Diccionario de la Real Academia Española (1869), con el objetivo de censurar el uso de un determinado provincialismo y recomendar su sustitución por un equivalente de uso registrado en dicho repertorio. Lo anterior se refleja fielmente en el comentario que el autor hace de 'coscacho'. En esta entrada, Rodríguez presenta como conclusión implícita que se trata de un uso censurable en el sentido de "golpe que se dá en la cabeza, que no saca sangre i duele" (Rodríguez, 1979 [1875]: 123). El dato es que, para nombrar este concepto, el Diccionario de la Real Academia Española registra el equivalente 'coscorrón'. Junto con esto, agrega el dato de que el uso de 'coscacho' significando ‘coscorrón' es exclusivo de Chile y Bolivia, o sea, es un provincialismo. La conclusión implícita se reafirma cuando dice "¿Por qué lo llamamos nosotros ‘coscacho’? Averígüelo Vargas!” (Rodríguez, 1979 [1875]: 123), frase con la cual deja el uso provincial y ajeno a la norma académica en el ámbito de lo irracional e inexplicable.

\subsubsection{ES CENSURABLE PORQUE NO HA SIDO USADO POR AUTORES LITERARIOS ESPAÑLES}

En tercer lugar, Rodríguez afirma que determinados provincialismos utilizados en el español chileno son censurables por no haber sido utilizados por los autores literarios españoles. Tal es el caso de 'con eso', monografía en la cual Rodríguez señala como conclusión implícita que el uso de esta locución es censurable basándose en el dato de que no recuerda haberla visto usada por los autores literarios españoles como equivalente de 'a fin de que' o 'con el objeto de que', como es 'tan corriente en Chile' (aporta dos ejemplos chilenos). La creencia normativa que se encuentra tras esta monografía implica que el uso que hacen los autores españoles de la lengua castellana es autorizado y correcto, entendiendo por autorizado aquel uso que se encuentra documentado en la literatura española, y que es correcto en la medida en que responde a un uso cultivado y culto de la lengua castellana. Cabe comentar que cuando Rodríguez habla de 'los autores españoles', se refiere a un determinado círculo de escritores que cita en su diccionario, y que son parte de sus lecturas personales. Entre ellos se encuentran Fernando de Rojas, Francisco de Quevedo, Luis Mariano de Larra, Fray Luis de Granada, Leandro Fernández de Moratín, Don Juan Manuel, Miguel de Cervantes, Pedro Calderón de la Barca y Tirso de Molina, entre otros. 


\subsubsection{ES CENSURABLE PORQUE ES UN EXTRANJERISMO SUSTITUIBLE POR UN TÉRMINO CASTIZO}

En cuarto lugar, Rodríguez censura el uso de extranjerismos que poseen equivalentes de uso en la lengua castellana. Tal es el caso de 'pasable', monografía en la cual el autor se limita a citar, a manera de dato, la opinión de dos autoridades americanas en materia idiomática: Rufino José Cuervo ("que tiene mui buenas narices") y Rafael María Baralt ("que no se anda con chicas"), para censurar el uso de esta unidad léxica. Dichos autores han censurado previamente en sus obras normativas el uso de 'pasable' en la lengua española hablada en América, por ser un extranjerismo de estirpe francesa ("solo lo emplean los mas desaforados galiparlistas", en palabras de Baralt), y han recomendado su sustitución por el equivalente castizo 'pasadero'. Los datos anteriores sirven de justificación a Rodríguez para aseverar que el uso de 'pasable' con el mismo significado que 'pasadero', 'no merece ser absuelto' de su erradicación del uso chileno. Resulta interesante cómo el autor a través de dos argumentos de autoridad como lo son la cita a Rufino José Cuervo y Rafael María Baralt, logra entregar mayor fuerza a su aseveración, dejando de lado toda explicación adicional.

\subsubsection{ES CENSURABLE PORQUE ES AJENO A LAS REGLAS GRAMATICALES DEL IDIOMA}

En quinto lugar, Rodríguez va a censurar provincialismos que atenten contra las reglas y preceptos de la gramática. En el prólogo de su obra, Rodríguez alude al modo en que este entiende la gramática: "el arte de hablar y escribir correctamente el español” (1979 [1875]: vii). Sabemos también, a partir del prólogo de su obra y de las fuentes que menciona en diversas entradas, que las gramáticas utilizadas por el autor fueron la de Bello, la de la Real Academia Española, la de Vicente Salvá y la de José Ramón Saavedra. Lo anterior se puede apreciar en la monografía de 'paquete', donde se censura la construcción 'mui paquete'. Para Rodríguez, el uso de esta estructura con el significado de 'peripuesto' es una falta a los preceptos consignados en la Gramática de la Real Academia Española, puesto que, según dicha obra, esta unidad léxica corresponde categorialmente a un sustantivo, y en Chile es utilizada incorrectamente como adjetivo. Junto con esto, alude a la transgresión al Diccionario académico que esto conlleva, puesto que allí la unidad léxica se registra como sustantivo, tal como debe utilizarse. Finalmente, destaca que corresponde a un uso exclusivo y propio de Chile.

\subsection{Clases de datos en las refutaciones}

\subsubsection{ES ACEPTABLE PORQUE LO REGISTRA EL DRAE Y EN REALIDAD NO ES PROVINCIAL}

En primer lugar, Rodríguez señala que ciertos usos considerados como provincialismos, no son tales si es que ellos se encuentran registrados en el Diccionario de la Real Academia Española (1869) sin la marca de provincial. Tal es el caso de la 
entrada 'Pancho', en donde Rodríguez concluye que este hipocorístico no merece ser calificado de provincialismo (es aceptable, por lo tanto), de acuerdo con el dato de que "viene [...] sin la nota de provincial en el Diccionario de la Academia" (Rodríguez, 1979 [1875]: 345). Además de este dato, Rodríguez afirma que Salvá ha declarado que 'Pancho' es un provincialismo de origen cubano, pero esta fuente pierde relevancia frente a la autoridad idiomática del Diccionario académico.

\subsubsection{ES ACEPTABLE PORQUE LO USAN AUTORIDADES LITERARIAS (ESPAÑOLAS O AMERICANAS)}

En segundo lugar, Rodríguez afirma que un uso supuestamente provincial no lo es en la medida en que este sea utilizado por autores literarios de la lengua castellana, sean peninsulares o americanos. En el caso de 'pensamiento', Rodríguez concluye que es un uso aceptable por no ser un provincialismo, puesto que, aunque no se encuentre registrado en el Diccionario de la Academia con el significado que se le da en Chile (equivalente de 'trinitaria'), cuenta con el dato de que es utilizado por importantes representantes de la literatura española y, por tanto, representantes del uso culto y correcto de la lengua española, sean estos escritores de origen americano o peninsular. Aporta como respaldos para este dato citas de la poetisa colombiana Agripina Samper de Ancízar y del poeta venezolano José Heriberto García de Quevedo. Cabe señalar que, en este caso, el argumento de autoridad del Diccionario académico queda desplazado por el argumento de autoridad relativo al uso de los escritores literarios. Sin embargo, añade una referencia al Diccionario Nacional o gran diccionario clásico de la lengua española (1846-1847), del español Ramón Joaquín Domínguez, que registra este uso sin marca provincial.

\subsubsection{ES ACEPTABLE PORQUE SATISFACE UNA NECESIDAD DENOMINATIVA}

En tercer lugar, Rodríguez tolera la existencia de provincialismos en la lengua estándar nacional si estos aluden a un referente o concepto para cuya denominación la lengua castellana no posee ningún equivalente exacto. Lo anterior se refleja claramente en la entrada de 'pirca', préstamo que "en quichua i araucano significa 'pared”' (Rodríguez, 1979 [1875]: 378). En Chile, 'pirca' se utiliza para aludir a la pared que se construye poniendo piedras brutas unas sobre otras, sin unirlas con nada o haciéndolo con barro, y que generalmente tienen un metro o metro y medio de altura. El dato que sirve a Rodríguez para concluir que es un uso aceptable consiste en que existe una laguna denominativa en la lengua castellana con respecto a este referente, ya que para llamar a este tipo específico de pared no existe otro equivalente exacto. Consecuentemente, un provincialismo que satisfaga dicho vacío, como 'pirca', será considerado por el autor como una 'voz útil', pues su adición al caudal léxico español responde a una necesidad denominativa. 
En cuartolugar, Rodríguez acepta en la lengua estándar nacionallos provincialismos que surgieron a partir de las reglas de derivación de la lengua castellana, o que se adecúan a ella, tal como estas se explican en las gramáticas de la lengua española conocidas. Tal es el caso de la entrada 'papa, papal, papero', en que se comenta el uso de estos provincialismos, de los cuales comentaremos solo 'papal'. La conclusión de Rodríguez es que es aceptable llamar 'papal' al sitio sembrado de papas a pesar de que esta palabra no se encuentre registrada en el Diccionario académico. El dato consiste en que 'papal' es una palabra de formación irreprochable, tal como Vicente Salvá en su Gramática ha señalado acerca de las palabras terminadas en '-al' para nombres colectivos, y J. Gómez Hermosilla en su Arte de hablar en prosa i verso. De acuerdo con el respaldo de estas autoridades, 'papal' corresponde a las reglas de la analogía de la lengua española, o, en palabras de Gómez Hermosilla, al 'genio' del idioma. Por otro lado, no menos importante, para Rodríguez, es que la palabra base del derivado, 'papa', tenga amplio uso en América.

\subsection{Discusión: Datos, garantías y creencias normativas}

Debemos destacar que las condiciones de aceptabilidad identificadas no sirven para Rodríguez como criterios normativos de manera aislada, sino que funcionan de manera concurrente. Es decir, no siempre es suficiente que un término, por ejemplo, posea una característica que lo haga inaceptable o aceptable normativamente, sino que además el autor sopesa si es que tiene otros méritos o defectos. Es lo que ocurre en el caso de 'chancaca', palabra que, a pesar de no estar incluida en el Diccionario académico a la fecha en que escribe Rodríguez, es considerada implícitamente por este autor como aceptable porque tiene un uso generalizado en América, incluso entre la gente culta, se entiende. Sin embargo, esto no anula la condición de que, en principio, son mejores las palabras que están incluidas en el Diccionario. Lo que se deduce de las palabras del autor es que en algún momento, piensa él, dicho vocablo tendrá que registrarse en el repertorio léxico oficial.

Algunas de las clases de datos que identificamos y analizamos por separado para las comprobaciones y las refutaciones corresponden a una sola creencia/garantía, pues varias de ellas muestran nada más que sus facetas positiva y negativa en los distintos tipos de evaluación. La clase 2 de las comprobaciones, ES CENSURABLE PORQUE EL DRAE YA REGISTRA OTRO TÉRMINO EQUIVALENTE QUE NO ES PROVINCIALISMO, encuentra su contraparte en la clase 3 de las refutaciones, Es ACEPTABLE PORQUE SATISFACE UNA NECESIDAD DENOMINATIVA. Estos patrones reflejan una sola creencia/garantía, que puede formularse del siguiente modo: Los USOS PROVINCIALES SON ACEPTABLES SOLO SI SIRVEN PARA DENOMINAR ALGO QUE NO TENGA YA UN NOMBRE REGISTRADO POR EL DRAE. Por otra parte, la clase 3 de las comprobaciones, Es CENSURAbLE PORQUe NO HA SIDO USADO POR AUTORES LITERARIOS ESPAÑOLES y la clase 2 de las refutaciones, 
Es ACEPTABLE PORQUE LO USAN AUTORIDADES LITERARIAS (ESPAÑOLAS O AMERICANAS), se pueden resumir en la creencia/garantía Los usos EMPLEADOS POR ESCRITORES PRESTIGIOSOS DE HABLA HiSPANA SIEMPRE SON LEGítimos. Finalmente, la clase 5 de las comprobaciones, Es CENSURABLE PORQUe ES AJENO A LAS REgLAS GRAMATICALES DEL IDIOMA y la categoría 4 de las refutaciones, Es ACEPTABLE PORQUE SE AJUSTA A LAS Reglas gramaticales del idioma, son dos caras de la garantía El uso legítimo SE AJUSTA A LAS REGLAS GRAMATICALES, SEGÚN ESTAS SE ENCUENTRAN CODIFICADAS EN LAS OBRAS ACADÉMICAS.

Dos de estas garantías reflejan el papel central que ha ocupado y ocupa hasta hoy la Real Academia Española en la codificación del español. Como consecuencia de la autoridad concedida oficialmente a esta institución desde su creación en el siglo XVIII (Lara, 2009b), se ha transformado en el principal agente estandarizador del idioma, tanto en España como en América. Por esta razón, sus obras codificadoras, Diccionario, Gramática y Ortografía, se erigen como encarnaciones de la lengua misma y, consecuentemente, como obras de autoridad indiscutida. Esta actitud hacia las obras académicas se refleja muy claramente, por ejemplo, en la idea de que el español legítimo es el que se encuentra registrado en el Diccionario de la Real Academia Española. En la época, el Diccionario de la Academia constituía el referente de máxima autoridad en asuntos de ejemplaridad idiomática en el mundo hispanohablante, ya que se pensaba que en él se registraba la lengua castellana originaria, primera, castiza, y correcta, de acuerdo con como había sido utilizada por los grandes autores de la literatura española del Siglo de Oro. Esta concepción del Diccionario académico es sin duda herencia del simbolismo que poseía para la lengua española el Diccionario de autoridades (Real Academia Española, 1726-1739), el cual tenía como objeto fijar la lengua española correcta documentando todos los usos léxicos de la lengua literaria, posicionando, por tanto, al uso literario como norma y ejemplo (Ruhstaller, 2003). Esto concuerda, por otra parte, con el valor simbólico que el diccionario monolingüe ha tenido en las comunidades europeas modernas desde el Renacimiento, en cuanto representante de la lengua legítima (Lara, 1997), o, como plantea Seargeant (2011), en cuanto encarnación del 'ideal platónico de diccionario' que contiene un registro autorizado y cabal del lenguaje y por tanto sirve como autoridad indiscutible para asuntos lingüísticos.

La otra garantía reflejada en dos clases de datos revela la importancia de la literatura en la estandarización del español. Su importancia se debe a que en dicho proceso los hispanohablantes, desde el Renacimiento, siguieron como modelo a los romanos, quienes a su vez habían continuado la tradición helénica de poner a la literatura como centro del cultivo planificado de la lengua (Clackson \& Horrocks, 2007). A partir de la argumentación de Dante en De vulgari eloquentia, según la cual las lenguas vulgares europeas debían seguir el modelo de los clásicos para obtener el nivel requerido por una lengua asociada a un Imperio o Estado, se consideró que la única variedad digna de ser sometida al arte que aseguraría su perpetuidad era la lengua literaria, la que serviría además en el futuro como instrumento de celebración de la gloria de las 
nuevas entidades políticas (Lara, 1997). Es esta idea, como ya hemos dicho, lo que sustenta la codificación lexicográfica del español: las autoridades del Diccionario de autoridades son los autores literarios, convertidos en tales sobre la base del concepto latino de la auctoritas. La importancia de la literatura está presente también con mucha fuerza en el diccionario de Rodríguez; de hecho, la mayoría de las citas que Rodríguez usa para autorizar o desligitimar usos provinciales son de escritores. Esto concuerda, además, con el papel principal que ocupa la literatura en las ideas lingüísticas de otros intelectuales influyentes en el Chile decimonónico, como Bello o Lastarria (Gallardo, 1988; Torrejón, 1989).

Solo tres clases de datos tienen relación unívoca con una garantía. La clase 1 de las comprobaciones, Es CENSURABLE PORQUE ESTÁ ASOCIADO PRINCIPALMENTE AL VULGO, refleja una idea elitista del uso aceptable, que tiene antecedentes en la idea de Andrés Bello de que el habla modélica es el de las personas educadas (Bello, 1847) y tiene sentido en el marco de la ideología racionalista de la época independentista, en que la educación era un valor que se tenía en alta estima, por lo cual la calidad de los hechos idiomáticos no podía medirse con la vara del uso de las personas que carecían de ella. Podría aventurarse que de algún modo se relaciona, como contraparte, con la garantía que señala que el uso de los autores literarios siempre es legítimo, si se entiende que los escritores representan un grado máximo de educación. La clase 4 de las comprobaciones, Es CENSURABLE PORQUE ES UN EXTRANJERISMO SUSTITUIBLE POR UN TÉRMINO CASTIZO, por su parte, responde al espíritu purista que impregnaba la ideología lingüística hispánica desde hace un par de siglos atrás (y de la mayoría de las lenguas europeas; Brunstad, 2003), mediante el rechazo de los elementos lingüísticos extranjeros, especialmente franceses, en el siglo xix. Por último, la clase 1 de las refutaciones, Es ACEPTABLE PORQUE LO REGISTRA EL DRAE Y EN REALIDAD NO ES PROVINCIAL, tiene carácter más bien técnico (o metodológico): se trata de que quien propuso originalmente que el uso comentado era incorrecto cometió un error al levantar sus datos. Sin embargo, refleja una garantía de orden más general, que señala que los usos provinciales son censurables si no cumplen con otros requisitos, como los que se señalan en las demás garantías. Rodríguez (Rodríguez, 1979 [1875]: 230), de hecho, llega a plantear abiertamente este criterio: “... la jente educada no debe hacer uso de provincialismos, sino en casos mui bien justificados". Esta misma idea es la que atraviesa, como dijimos, al género completo de los diccionarios de provincialismos.

En resumen, las creencias lingüísticas normativas que subyacen, a modo de garantías, a la argumentación de Rodríguez en su Diccionario son las siguientes:

1. Necesidad denominativa: Los usos PROVINCIALES SON ACEPTABLES SOLO SI SIRVEN PARA DENOMINAR ALGO QUE NO TENGA YA UN NOMBRE REGISTRADO POR EL DRAE.

2. Autoridad literaria: Los usos EMPLEAdos POR ESCRITORES PRESTIGIOSOS DE HABLA HISPANA SIEMPRE SON LEGÍTIMOS. 
3. Educación: El uSo LEgítimo ES EL DE LAS PERSONAS EDUCADAS.

4. Ajuste a reglas gramaticales codificadas: EL USO LEGítimo SE AJUSTA A LAS REGLAS GRAMATICALES, SEGÚN ESTAS SE ENCUENTRAN CODIFICADAS EN LAS OBRAS ACADÉMICAS.

\section{Diccionario: Los usos legítimos son los registrados en el Diccionario ACADÉMICO.}

6. Purismo: Los usos Lingüísticos DE ORIGEN EXTRANJERo (ESPECIALMENTE LOS FRANCESES) NO SON ACEPTABLES.

7. Antiprovincialismo: Los usOS PROVINCIALES, EN PRINCIPIO (ES DECIR, SI NO
CUMPLEN ALGUNA DE LAS CARACTERÍSTICAS ANTERIORES), SON INAPROPIADOS.

\section{CONCLUSIONES}

En el presente estudio nos propusimos observar cuáles eran, en el Chile de fines del siglo XIX, las creencias que funcionaban como criterios normativos en la estandarización lingüística a través del discurso argumentativo contenido en el género lexicográfico de los diccionarios de provincialismos, tal como se encuentran representadas en la obra de Rodríguez (1979 [1875]). El modelo de análisis argumentativo de Toulmin (2007) mostró ser útil para develar siete de estas creencias o criterios. Estas creencias muestran un modelo ideal de lengua española de acuerdo con el cual esta lengua ya había alcanzado un grado alto y más que suficiente de estandarización y, por tanto, debía estar cerrada a las innovaciones o préstamos innecesarios (como puede apreciarse en las creencias que etiquetamos como 'necesidad denominativa', 'ajuste a reglas gramaticales codificadas', 'diccionario' y 'purismo', que muestran además el papel central que la Real Academia Española ha tenido en esta estandarización), además de ser geográficamente homogénea ('antiprovincialismo') y cuya calidad se entendía sobre el trasfondo de la literatura ('autoridad literaria') y fundamentada en el grado de educación de sus usuarios ('educación').

Esta visión de la lengua es congruente con la ideología monoglósica (es decir, contraria a la diversidad lingüística y al multilingüismo) y purista que ha caracterizado a la cultura lingüística hispánica durante los últimos siglos (Del Valle \& GabrielStheeman, 2002), en particular la propugnada por las Academias de la Lengua Española, y que es especialmente compatible con la ideología lingüística de los unionistas chilenos, quienes tomaban como modelo idiomático, para la constitución de la lengua estándar nacional, la variedad peninsular metropolitana, codificada en las obras académicas (Diccionario y Gramática de la Real Academia Española) y manifestada principalmente en el uso de los escritores castellanos. Los unionistas, además, tenían una concepción racionalista del proceso de estandarización lingüística (Geeraerts, 2006) en el sentido de que concebían que era necesario mantener la 
lengua española unida y homogénea e instrumentalizarla en la 'difusión de las luces', tal como lo habían hecho los revolucionarios franceses en su propio contexto lingüístico (Wright, 2011). La actitud lingüística basada en este elemento ideacional evalúa favorablemente la variedad codificada en las obras académicas (Diccionario y Gramática de la Real Academia Española) mientras que evalúa negativamente el elemento provincial, al que se le exige cumplir con ciertos requisitos para llegar a ser considerado admisible.

Para finalizar, deseamos llamar la atención sobre el interés que tendría comparar en investigaciones futuras las ideas de Rodríguez con las que otros autores de gramáticas y diccionarios manifiestan durante la época en Chile, con el fin de identificar convergencias y divergencias. Igualmente, queremos destacar la pertinencia de estudiar cómo estas normas, criterios y creencias se proyectaban en una dirección argumentativa de carácter dialógico, en lo que Andersen (2009) denomina 'negociación de normas lingüísticas'. Este mecanismo podría estudiarse, por ejemplo, mediante el examen del tejido intertextual que la obra de Rodríguez establece con sus propias fuentes y con comentaristas como Solar (1876) y Paulsen (1876), así como diccionaristas posteriores. El análisis de la negociación de normas lingüísticas en el Chile del siglo XIX permitiría apreciar el proceso de estandarización y los criterios normativos de una manera dinámica y emergente. 


\section{REFERENCIAS BIBLIOGRÁFICAS}

Albarracín, D., Zanna, M. P., Johnson, B. T. \& Kumkale, G. T. (2005). Attitudes: Introduction and scope. En D. Albarracín, B. T. Johnson \& M. P. Zanna (Eds.), The Handbook of Attitudes (pp. 3-19). Mahwah/Londres: Lawrence Erlbaum.

Andersen, H. (2009). Living norms. En I. Lunde \& M. Paulsen (Eds.), Poets to Padonki: Linguistic Authority \& Norm Negotiation in Modern Russian Culture (pp. 18-33). Bergen: Universidad de Bergen.

Becerra, Y., Castro, V. \& Garrido, C. (2007). Tres repertorios léxicos diferenciales del español de Chile en el siglo xix. Un estudio metalexicográfico. Tesis de licenciatura, Universidad de Chile, Santiago, Chile.

Bello, A. (1847). Gramática de la lengua castellana destinada al uso de los americanos. Santiago: Imprenta del Progreso.

Bizer, G. (2004). Attitudes. En Ch. Spielberger (Ed.), Encyclopedia of Applied Psychology (pp. 245-249). Londres: Elsevier Academic Press.

Blanco, M. (1875). Cartas de Don Manuel Blanco Cuartín a don Zorobabel Rodríguez sobre Chilenismos. Santiago: El Mercurio.

Blommaert, J. (1999). The debate is open. En J. Blommaert (Ed.), Language Ideological Debates (pp. 1-38). Berlín/Nueva York: Mouton de Gruyter.

Brunstad, E. (2003). Standard language and linguistic purism. Sociolinguistica, 17, 5270.

Castillo, N. (1995). Elprimer diccionario de chilenismos: Aproximación metalexicográfica. Tesis de licenciatura, Pontificia Universidad Católica de Chile, Santiago, Chile.

Chávez, S. (2009). Diccionarios del español de Chile en su fase precientífica: Un estudio metalexicográfico. Tesis de magíster, Universidad de Chile, Santiago, Chile.

Chávez, S. (2010). Ideas lingüísticas en prólogos de diccionarios diferenciales del español de Chile. Etapa 1875-1928. Boletín de Filología, XLV(2), 49-69.

Clackson, J. \& Horrocks, G. (2007). The Blackwell History of the Latin Language. Malden: Blackwell.

Coleman, J. \& Ogilvie, S. (2009). Forensic Dictionary Analysis: Principles and practice. International Journal of Lexicography, 22(1), 1-22.

Correa, S. (1997). Zorobabel Rodríguez, católico liberal. Estudios Públicos, 66, 387-426. 
Coupland, N. \& Jaworski, A. (2004). Sociolinguistic perspectives on metalanguage: Reflexivity, evaluation and ideology. En N. Coupland, A. Jaworski \& D. Galasinski (Eds.), Metalanguage: Social and Ideological Perspectives (pp. 15-51). Berlín: Mouton de Gruyter.

De los Heros, S. (2012). Utopia y realidad. Nociones sobre el estándar lingüistico en la esfera intelectual y educativa peruana. Madrid: Iberoamericana.

Del Valle, J. \& Gabriel-Stheeman, L. (2002). Nationalism, hispanismo and monoglossic culture. En J. del Valle \& L. Gabriel-Stheeman (Eds.), The Battle over Spanish between 1800 and 2000. Language ideologies and Hispanic intellectuals (pp. 1-13). Londres/Nueva York: Routledge.

Gallardo, A. (1988). Un aspecto del desarrollo de la identidad lingüística chilena: José Victorino Lastarria y el Movimiento Literario de 1842. Revista de Lingüistica Teórica y Aplicada, 26, 29-40.

Kroskrity, P. V. (2010). Language ideologies -Evolving perspectives. En J. Jaspers, J. O. Östman \& J. Verschueren (Eds.), Society and Language Use (pp. 192-211). Amsterdam/Philadelphia: John Benjamins.

Garrett, P. (2010). Attitudes to language. Cambridge: Cambridge University Press.

Geeraerts, D. (2006). Cultural models of linguistic standardization. En D. Geeraerts (Ed.), Words and other wonders. Papers on lexical and semantic topics (pp. 272-306). Berlín/Nueva York: Mouton de Gruyter.

Haensch, G. (2000). Introducción. En Textos clásicos para la historia de la lexicografía del español en América [CD-ROM]. Versión 1.1. Madrid: Fundación Histórica Tavera/Fundación MAPFRE.

Inoue, M. (2006). Standardization. En K. Brown (Ed.), Encyclopedia of Language \& Linguistics (pp. 121-127). Oxford: Elsevier.

Joseph, J. E. (1987). Eloquence and power. The rise of language standards and standard sanguages. Londres: Frances Pinter.

Lara, L. F. (1997). Teoría del diccionario monolingüe. México, D. F.: El Colegio de México.

Lara, L. F. (2009a). Por una reconstrucción de la idea de la lengua española. Más allá de las fronteras instituidas. En J. del Valle (Ed.), Lengua histórica y normatividad (pp. 157-193). México, D. F.: El Colegio de México.

Lara, L. F. (2009b). Normas lingüísticas: Pluralidad y jerarquía. En L. F. Lara (Ed.), Lengua histórica y normatividad (pp. 47-69). México, D. F.: El Colegio de México. 
Maio, G. R., Olson, J. M., Bernard, M. M. \& Luke, M. A. (2006). Ideologies, values, attitudes, and behavior. En J. Delamater (Ed.), Handbook of Social Psychology (pp. 283-308). Nueva York: Springer.

Marafioti, R. (2005). Los patrones de la argumentación: La argumentación en los clásicos y en el siglo xx. Buenos Aires: Biblos.

Martínez de Sousa, J. (1995). Diccionario de lexicografía práctica. Barcelona: Biblograf.

Matus, A. (1994). Períodos en la lexicografía diferencial del español de Chile. En Actas del X Congreso de la Asociación de Academias de la Lengua Española (pp. 189-199). Madrid: Real Academia Española/Espasa Calpe.

Matus, A., Dargham, S. \& Samaniego, J. L. (1992). Notas para una historia del español en Chile. En C. Hernández (Coord.), Historia y presente del español de América (pp. 543-564). Valladolid: Junta de Castilla y León/PABECAL.

Metzeltin, M. (2011). La construcción discursiva de la República de Chile. Boletín de Filología, XLVI(1), 239-253.

Moré, B. (2002). The ideological construction of an empirical base. Selection and elaboration in Andrés Bello's grammar. En J. del Valle \& L. Gabriel-Stheeman (Eds.), The Battle over Spanish between 1800 and 2000. Language ideologies and Hispanic intellectuals (pp. 42-63). Londres/Nueva York: Routledge.

Quesada, M. Á. (2002). El español de América: Historia de un concepto. En M. A. Quesada (Ed.), El español de América (pp. 15-39). Cártago: Editorial Tecnológica de Costa Rica.

Paulsen, F. (1876). Reparo de reparos, o sea, ligero examen de los «Reparos al "Diccionario de Chilenismos" de don Zorobabel Rodríguez», por don Fidelis P. del Solar. Santiago: Chile.

Preston, D. (2011). Methods in (applied) folk linguistics. AIL A Review, 24, 15-39.

Real Academia Española (1726-1739). Diccionario de la lengua castellana, en que se explica el verdadero sentido de las voces, su naturaleza y calidad, con las phrases o modos de hablar, los proverbios o refranes, y otras cosas convenientes al uso de la lengua. Madrid.

Real Academia Española (1869). Diccionario de la lengua castellana por la Real Academia Española. Madrid.

Rodríguez, Z. (1979 [1875]). Diccionario de chilenismos. Valparaíso: EUV.

Rojas, D. (2010). Estandarización lingüística y pragmática del diccionario: Forma y función de los "diccionarios de provincialismos" chilenos. Boletín de Filología, XLV(1), 209-233. 
Ruhstaller, S. (2003). Las obras lexicográficas de la Academia. En M. A. Medina Guerra (Coord.), Lexicografía española (pp. 235-261). Barcelona: Ariel.

Seargeant, P. (2011). Lexicography as a philosophy of language. Language Sciences, 33, $1-10$.

Solar, F. (1876). Reparos al "Diccionario de chilenismos" del señor don Zorobabel Rodríguez: Santiago: Chile.

Toulmin, S. (2007). Los usos de la argumentación. Barcelona: Ediciones Península.

Torrejón, A. (1989). Andrés Bello, Domingo Faustino Sarmiento y el castellano culto de Chile. Thesaurus, XLIV(3), 534-558.

Woolard, K. A. (1998). Introduction: Language Ideology as a Field of Inquiry. En B. B. Schieffelin, K. A. Woolard \& P. V. Kroskrity (Eds.), Language Ideologies. Practice and Theory (pp. 3-47). Oxford: Oxford University Press.

Wright, S. (2011). Language and nation building in Europe. En B. Kortmann \& J. van der Auwera (Eds.), The languages and linguistics of Europe: A comprehensive guide, (pp. 775-788). Berlín/Boston: Walter de Gruyter.

\section{NOTAS}

1 Financiado por Programa U-APOYA Línea 2: Concurso de Proyectos de Investigación en Ciencias Sociales, Humanidades, Artes y Educación VID 2011; código SOC U-SOC-11/12; Universidad de Chile.

2 Esta y todas las demás citas de obras en inglés han sido traducidas al español por los autores del presente artículo.

3 Agradecemos las pertinentes sugerencias y comentarios de dos evaluadores anónimos, quienes contribuyeron a mejorar sustancialmente la calidad de este trabajo.

4 Usamos 'monografía' como equivalente de 'artículo lexicográfico', de acuerdo con la terminología metalexicográfica de la Escuela de Ausburgo (Martínez de Sousa, 1995: s. v. monografía). 